\title{
Viscoelastic effects on the dynamics of a rising bubble
}

\section{Woodrow L Shew and Jean-François Pinton}

Laboratoire de Physique, CNRS and École Normale Supérieur de Lyon, Lyon 69007, France

E-mail:wlshew@ens-lyon.fr and pinton@ens-lyon.fr

Received 19 October 2005

Accepted 22 November 2005

Published 16 January 2006

Online at stacks.iop.org/JSTAT/2006/P01009

doi:10.1088/1742-5468/2006/01/P01009

\begin{abstract}
The dynamics of millimetre-sized air bubbles rising through a still $1 \%$ polyacrylamide/water solution are investigated using precise ultrasound velocity measurements combined with high-speed video. The oscillatory path dynamics are compared to the those of bubbles rising in water. We find distinctive changes in path geometry, the onset of path instability, as well as lift forces. Since the Deborah number for these dynamics is near unity, the observed effects are attributed to viscoelastic effects in the wake of the bubble.
\end{abstract}

Keywords: bubbles and drops, hydrodynamic instabilities 


\section{Contents}

1. Background 2

2. Experimental apparatus, methods, and fluids 3

3. Reference case: rising bubbles in water 6

4. Coordinate system and forces $\quad 7$

5. Experimental observations 9

6. Summary and discussion 13

$\begin{array}{ll}\text { Acknowledgments } & 14\end{array}$

References $\quad 14$

\section{Background}

The distinctive oscillatory path of a millimetre-sized bubble as it rises through still liquid has been a source of fascination for scientists since the time of Leonardo da Vinci [1]. A number of recent investigations have led to significant advances in the understanding of bubble path instabilities [2]-[11]. The inertia of such a bubble is much smaller than that of the surrounding liquid, making it a very sensitive probe of the hydrodynamic forces at work. Furthermore, since the boundary of a bubble is free-slip, the dynamics are governed by bulk fluid properties with nearly no influence of viscous boundary layers (e.g. [12]). The aim of the experiments presented here is to take advantage of these features of rising bubbles as well as a sensitive ultrasound velocimetry technique to explore the effects of a viscoelastic fluid medium on the path instability of a bubble.

Bubbles smaller than about $1 \mathrm{~mm}$ in radius rise in a straight path (e.g. $[13,14]$ ). For slightly larger bubbles, the path is initially straight but quickly becomes unstable. In a primary instability, the path begins to oscillate in a zigzag motion confined to a vertical plane. A secondary instability follows soon after, resulting in a spiralling path. Many investigators have contributed to the understanding of these path instabilities with experimental, numerical, and analytical efforts [2]-[11], [15]-[22]. This paper extends previous work of the authors, which addressed the dynamics of bubble path instability in water [2]. The oscillatory motions are caused by the development of a non-axisymmetric wake consisting of a pair of long thin vortices trailing the bubble. Experimental and numerical observations of these wake structures as well as bubble trajectories have clarified the role of the wake in path instability [2]-[11]. Using a high-speed camera and an ultrasound device for probing the bubble speed, we make precise three-dimensional measurements of bubble trajectories. By comparing the bubble path dynamics in a nonNewtonian fluid to those in water, we uncover viscoelastic effects in the bubbles' wakes.

In the next section we describe the experimental apparatus, fluid rheology and measurement techniques. Then we review the main features of the dynamics of bubbles rising in Newtonian fluids. Next, we define the coordinate system, equations of motion, 


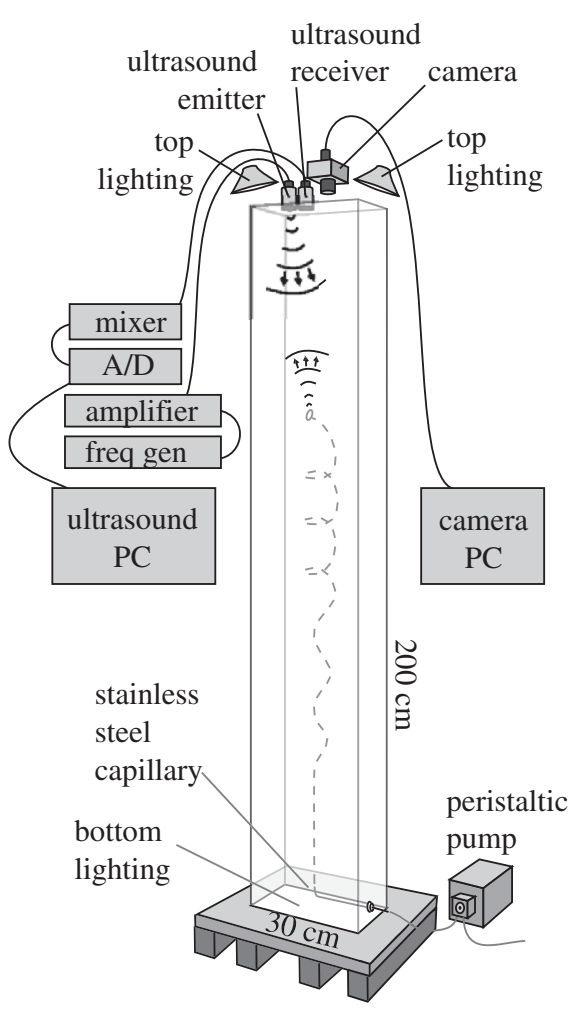

Figure 1. Schematic of experimental setup. As the bubble rises through $2 \mathrm{~m}$ its vertical velocity is measured using ultrasound and its horizontal position is obtained with a high-speed video camera.

and assumptions employed to extract knowledge of forces from the bubble trajectory. Experimental observations are presented in section 5 and finally interpreted in section 6 .

\section{Experimental apparatus, methods, and fluids}

Apparatus and methods. The experimental methods and apparatus are the same as those used in a previous study and are more thoroughly detailed there [2]. As illustrated in figure 1, the bubbles rise $2 \mathrm{~m}$ through a tank filled with $180 \mathrm{l}$ of either water or waterpolyacrylamide solution. The vessel is $30 \mathrm{~cm} \times 30 \mathrm{~cm}$ in horizontal dimensions and the bubble rises near the centre, avoiding any influence of the walls. Air bubbles are produced by pumping air through a small capillary tube at the bottom of the tank. Care was taken to allow the fluid at least $3 \mathrm{~min}$ to return to a quiescent state before releasing another bubble.

The variable parameter for these experiments is the size of the bubble. We measure the volume of each bubble individually. At the end of its ascent each bubble is trapped under a submerged plate at the top of the tank. Using a syringe, the bubble is then sucked into a transparent tube with known inner diameter (ID $0.51 \pm 0.005 \mathrm{~mm}$ ). With fluid before and after, the length of the air-plug formed by the bubble is measured with calipers $( \pm 0.2 \mathrm{~mm}$ precision) and used to calculate the volume. The radius of the bubble expands by about $6 \%$ during the rise due to the decrease in hydrostatic pressure. This 
expansion is accounted for in the calculations of forces and each instance where bubble size or Reynolds number is presented in this paper. The Reynolds number is defined as $R e=2 U R / \nu$, where $R$ is the radius of a sphere which has the same volume as that measured for the bubble. The velocity $U$ is the instantaneous speed of the bubble. The kinematic viscosity $\nu$ of the polymer solution depends weakly on the shear rate, but is close to $5 \%$ greater than that of water at the same temperature (see below for fluid rheology details). For our experiments we observed a range of bubble sizes $0.84 \mathrm{~mm}<R<1.25 \mathrm{~mm}$; the velocities were $32 \mathrm{~cm} \mathrm{~s}^{-1}<U<36 \mathrm{~cm} \mathrm{~s}^{-1}$, and kinematic viscosities $1.04 \times 10^{-6} \mathrm{~m}^{2} \mathrm{~s}^{-1} \leq \nu<1.06 \times 10^{-6} \mathrm{~m}^{2} \mathrm{~s}^{-1}$, yielding a range of Reynolds number, $500<R e<860$.

Our measurements of certain forces require knowledge of the bubble shape. We do not measure bubble shape in our experiments, but it has been shown in similar experiments that the bubble is very close to an oblate ellipsoid $[13,5,6,10]$. The aspect ratio $\chi$ of the bubble is defined as the semimajor axis length divided by the semiminor axis length. We use Duineveld's measurements [13] of $\chi$ for different bubble sizes to estimate $\chi$ for our bubbles. Justification of this method lies in the agreement of our data with drag theory, as will be shown in section 5 (see also [2]).

The trajectory of the bubble is deduced from measurements of the vertical component of the bubble velocity and the horizontal components of its position. The vertical velocity component is obtained using an ultrasound technique. One transducer continuously emits sound at $2.8 \mathrm{MHz}$ into the fluid. As the bubble rises the sound scattered from its surface is recorded by a second ultrasound transducer. A combination of digital signal processing and numerical algorithms is used to extract the Doppler-shifted frequency of the sound scattered from the bubble. This technique has been described in detail in previous publications [2], [23]-[25]. The component of the bubble's velocity along the line between the bubble and the ultrasound receiver is directly related to the Doppler frequency shift. Since the line between the bubble and the receiver oscillates as the bubble's path oscillates, an iterative correction algorithm is employed to obtain the true vertical component. A correction of about $4 \%$ is necessary when the bubble is within a few tens of centimetres from the receiver probe. The absolute and relative accuracy of the velocity measurement after correction are typically about $\pm 0.007 \mathrm{~m} \mathrm{~s}^{-1}$ and $\pm 0.0007 \mathrm{~m} \mathrm{~s}^{-1}$ respectively. The time resolution of these measurements is less than $1 \mathrm{~ms}$.

A high-speed video camera is used to obtain the horizontal motion of the bubble. The camera is positioned above the vessel close to the ultrasound receiving array so that its line of sight is aligned with the bubble's path. The camera is operated at 125 frames s${ }^{-1}$ and with $512 \times 512$ pixel resolution. The bubble position is extracted from movies using Matlab image processing routines.

Fluid properties. Two series of measurements were carried out. First, we observed the behaviour of 20 air bubbles rising in fresh tap water. Then, about 41 of water were removed and replaced with a 50/50 polyacrylamide/water solution. In the polymer solution, we recorded the trajectories of 30 rising bubbles. We note that both sets of experiments were carried out on the same day with the same water to assure that the comparison of the two data sets is most meaningful. Before filling, the vessel walls and all parts in contact with fluid were cleaned thoroughly with methanol and then rinsed with tap water for 5 min. The temperature of the fluid was $18.5 \pm 0.25^{\circ} \mathrm{C}$ for the water experiments and $19 \pm 0.25^{\circ} \mathrm{C}$ for the polymer solution. The effect of the temperature difference on the fluid viscosities is 

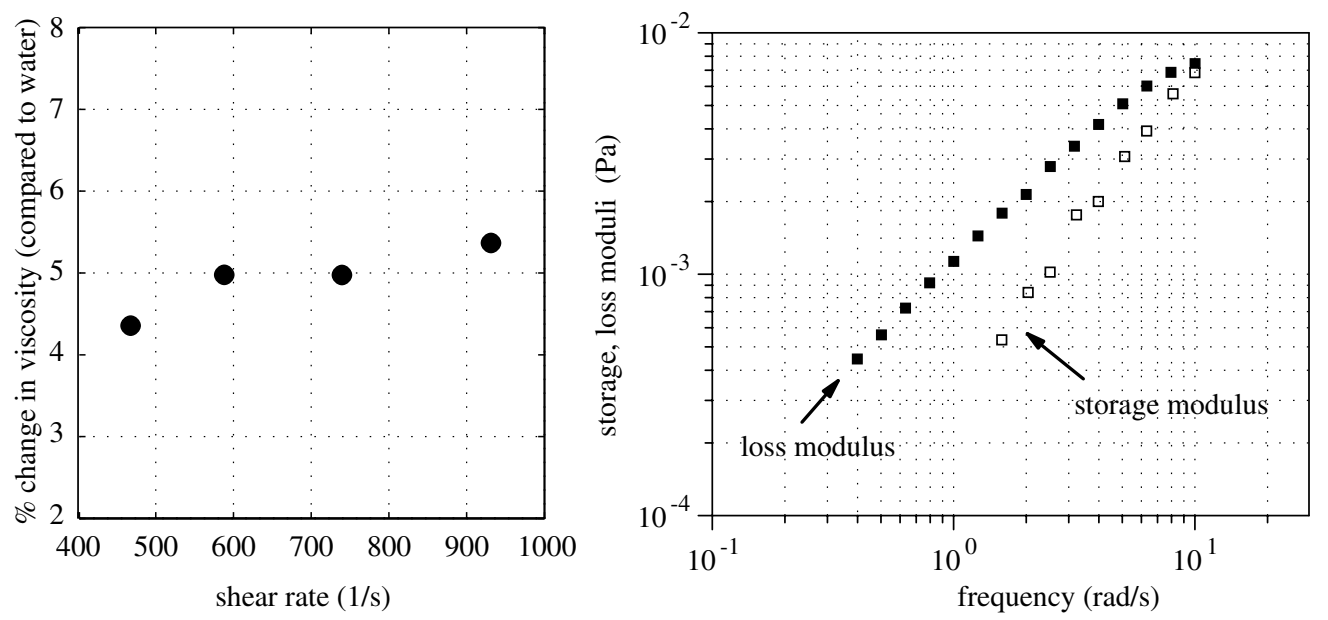

Figure 2. (a) Percentage change in viscosity compared to water as a function of shear rate. (b) Storage and loss moduli as a function of oscillatory frequency. Rheology measurements were performed by the research group of Professor WM Kulicke at the Institute of Technical and Molecular Chemistry, Hamburg University, Germany (see footnote 1).

accounted for in the measurements discussed in section 5. Furthermore, the temperature gradient was always less than $0.009^{\circ} \mathrm{C} \mathrm{cm}^{-1}$.

The polymer solution was composed of $1 \%$ by weight polyacrylamide and $99 \%$ water. The polyacrylamide was supplied by Sigma-Aldrich (reference number 434949 ) in a $50 \%$ by weight aqueous solution. The average molecular weight is $10^{4}$. The rheological properties of the polymer solution were tested directly by the research group of Professor WM Kulicke at the Institute of Technical and Molecular Chemistry, Hamburg University, Germany $^{1}$. Viscosity as a function of shear as well as loss modulus $G^{\prime \prime}$ and storage modulus $G^{\prime}$ were measured for a range of shear rates and oscillation frequencies. The results are shown in figure 2. The viscosity can attain values up to $5 \%$ more than that of water for the range of shear rates one might expect near a rising bubble. For low shear rates, one may estimate a relaxation time for the fluid based on simple fluid theory,

$$
\lambda_{\mathrm{r}} \approx \eta / G \sim 0.1 \mathrm{~s},
$$

where $G=\sqrt{G^{\prime 2}+G^{\prime \prime 2}}, \eta=\nu \rho$ is the viscosity, and $\rho$ is the density of the fluid (e.g. [26]). An upper bound for the timescale $\lambda_{\mathrm{r}}$ may also be estimated from the inverse of the shear rate for which $G^{\prime}$ equals $G^{\prime \prime}$. We may extrapolate the curves in figure 2 to estimate this intersection point between 10 and $100 \mathrm{rad} \mathrm{s}^{-1}$, yielding a timescale around $0.1-1 \mathrm{~s}$, consistent with the estimate above. As will be made clear in the next section, the timescale associated with the shear rate of the flow around the bubble is $\lambda_{\mathrm{f}}=D / U \sim 0.01 \mathrm{~s}$. The ratio of the fluid relaxation time to the flow timescale is the Deborah number, $D e=\lambda_{\mathrm{r}} / \lambda_{\mathrm{f}}$. Allowing for some decrease in $\lambda_{\mathrm{r}}$ at higher shear rates, we estimate $D e=1-10$. Although we do not measure the first normal stress difference $N_{1}$ for our fluid, models as well as empirical evidence suggest that a non-zero storage modulus implies a non-zero $N_{1}$

\footnotetext{
${ }^{1}$ W M Kulicke, Institute of Technical and Molecular Chemistry, Bundesstrasse 45, 20146 Hamburg, Germany.
} 

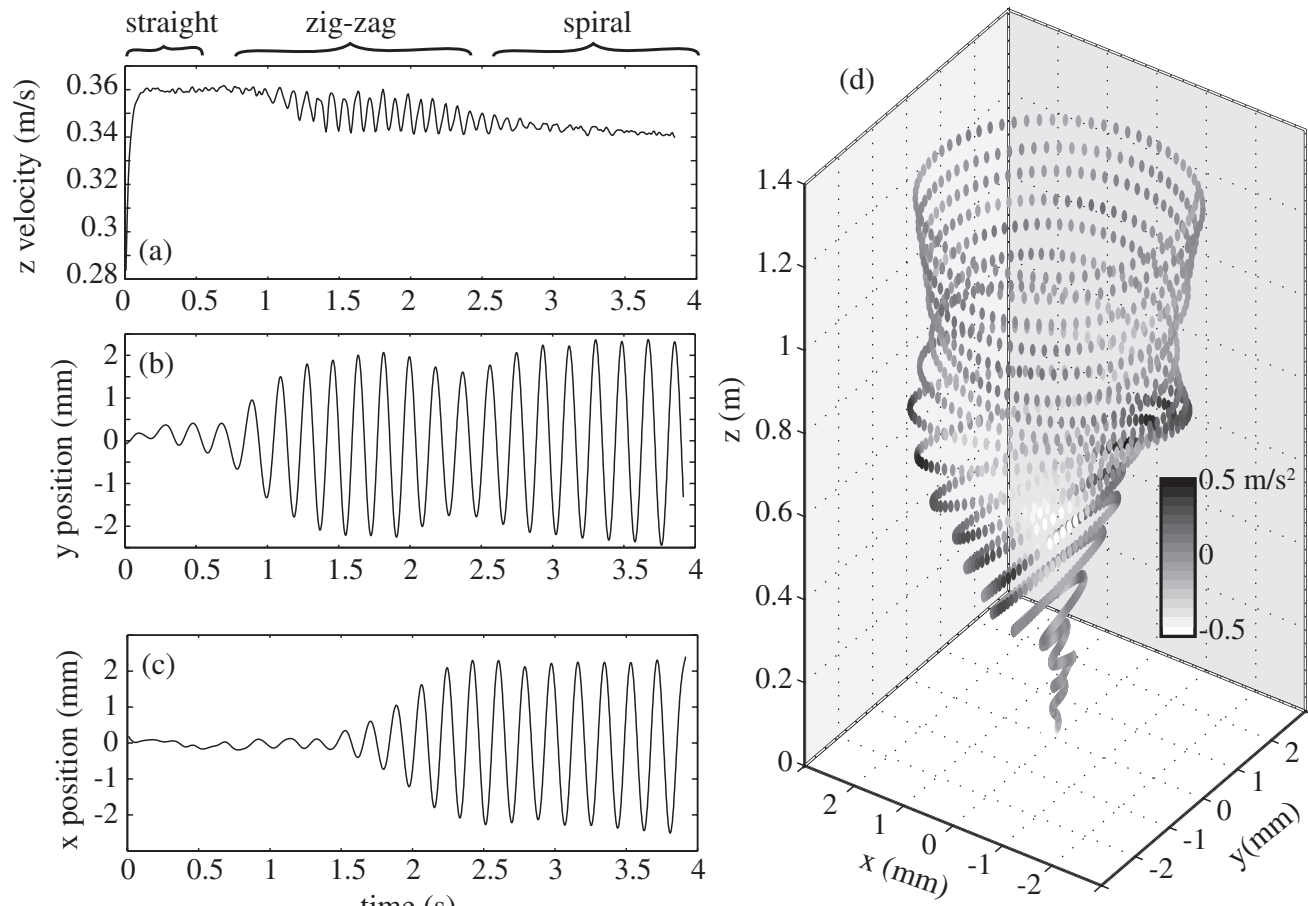

Figure 3. Example trajectory of a $1.12 \mathrm{~mm}$ radius (at $1 \mathrm{~atm}$ ) bubble in water $(R e=810)$. (a) Vertical component of velocity as determined from ultrasound technique, ((b), (c)) horizontal position from camera, and (d) threedimensional reconstruction of full trajectory with greyscale indicating magnitude of acceleration. The straight, zigzag, and spiral portions of the trajectory are labelled above the velocity time series (a). The same qualitative behaviour is observed in the polymer solution.

(e.g. $[27,28])$. For example, simple fluids theory gives $N_{1}=2 G^{\prime}$. At near unity Deborah numbers and with non-zero first normal stress difference one may expect to observe viscoelastic fluid dynamics (e.g. [29,33]).

\section{Reference case: rising bubbles in water}

An example trajectory of a $1.12 \mathrm{~mm}$ (at $1 \mathrm{~atm}$ ) radius bubble rising in water is shown in figure 3. We found that the trajectories of bubbles in our polymer solution are very similar to that depicted in figure 3. To establish a context for the results and comparisons in section 5, we will first review what is known about the dynamics of bubbles in water.

Figure 3 illustrates well the different types of motion that one may expect for bubbles in the size range we study. Upon release from the generating tube, the bubble accelerates from rest to a terminal speed around $36 \mathrm{~cm} \mathrm{~s}^{-1}$. A small enough bubble will continue to rise in a straight line at its terminal speed until it reaches the surface, but the paths of larger bubbles like the example in figure 3 become unstable and begin to zigzag. This first path instability manifests itself in velocity oscillations as well as oscillations in one, but not both, horizontal position coordinates [2, 5, 8, 6, 22]. The zigzag motion is confined to a vertical plane. Some cycles after the onset of the zigzag, the second horizontal 
position coordinate also begins to oscillate and the velocity gradually returns to a steady value, although lower than that of a straight rising bubble $[2,15,8]$. When the bubble has completed the transition from the zigzag to the spiral state the motion traces out a circle when projected onto a horizontal plane $[2,6,11]$. These dynamics are tied to hydrodynamic instabilities in the fluid around the bubble. We note that the period of both zigzag and spiral oscillations is about $0.2 \mathrm{~s}$, which is close to the estimated relaxation time for our polymer solution.

Let us also review what is known about the flow field surrounding the bubble. In particular, the wake of the bubble plays a significant role in the path dynamics. For a straight rising bubble the flow past the sides of the bubble, as well as the wake, is axisymmetric $[5,13,8,12]$. The transition to zigzag motion has been observed to coincide with a breakdown in axisymmetry. Flow is faster around one side of the bubble and a pair of thin streamwise vortices with opposite sense of rotation develop in the wake, similar to the trailing vortices behind an airplane $[5,8]$. The analogy to an airplane wing can be carried further. Like a wing, the fast flow on one side of the bubble results in a lower pressure region which provides a lift force on the bubble. From a complementary and consistent viewpoint, the wake vortices, like wing-tip vortices, tend to pump fluid from the fast side to the slow side of the bubble. The resulting momentum flux must be balanced by the motion of the bubble towards the fast side $[2,5,7,3,6,8]$. For zigzag motions the two trailing vortices are located symmetrically on either side of the zigzag plane. The vortices are displaced to one side for the spiralling motion.

\section{Coordinate system and forces}

In analytical investigations into bubble dynamics, potential flow theory is often successfully used to describe gross features, while regions of the flow with vorticity must also be accounted for to make precise predictions. Similarly, we will often divide our attention between irrotational and rotational regions of the flow in the discussions to follow. Kirchhoff's equations [30] for the motion of a rigid body in a purely irrotational flow have recently been generalized and successfully implemented for the case of viscous flow at high Reynolds number [31, 8, 32]. Although we do not measure our bubble shapes, other experiments have shown that for bubbles in the size range we investigate, the shape is nearly steady in time $[6,10]$. With this assumption, we may employ the generalized Kirchhoff equations,

$$
\begin{aligned}
& \mathbb{D} \frac{\mathrm{d} \boldsymbol{\Omega}}{\mathrm{d} t}+\boldsymbol{\Omega} \times \mathbb{D} \boldsymbol{\Omega}+\mathbf{U} \times \mathbb{A} \mathbf{U}=\boldsymbol{\Gamma}, \\
& \mathbb{A} \frac{\mathrm{d} \mathbf{U}}{\mathrm{d} t}+\boldsymbol{\Omega} \times \mathbb{A} \mathbf{U}=\mathbf{F} .
\end{aligned}
$$

Here the velocity $\mathbf{U}$ of the bubble's centre of mass and angular velocity $\Omega$ about its centre of mass are evaluated in an inertial reference frame (like our measurements) and then projected onto a coordinate system which rotates with the bubble. The coordinate system is defined as follows. The 1-direction is in the same direction as the velocity vector. The 2-direction is normal to the 1-direction and defined such that the 1-2 plane is vertical and contains the velocity vector. And the 3-direction is horizontal and normal to both the 1- and 2-directions. With these definitions a straight rising bubble moves only in the 1direction, a zigzag is confined to the 1-2 plane, and a spiral moves in all three dimensions. 


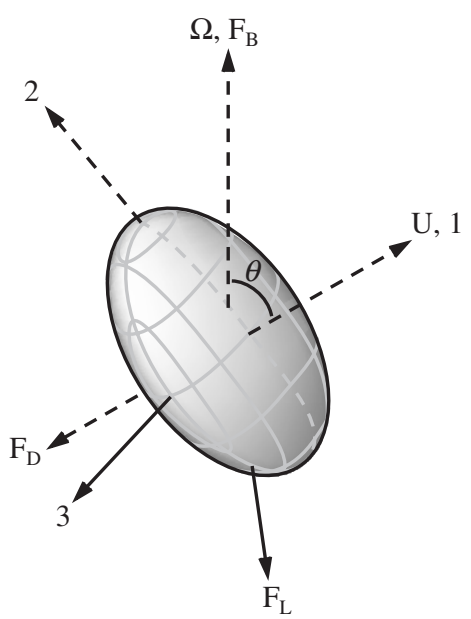

Figure 4. Diagram of the coordinate system, velocity $U$, angular velocity $\Omega$, pitch angle $\theta$, and external forces $\left(F_{\mathrm{B}}, F_{\mathrm{D}}, F_{\mathrm{L}}\right)$ present for a spiralling bubble. The dashed lines lie in the $1-2$ plane.

In this coordinate system, the added mass and added rotational inertia tensors, $\mathbb{A}$ and $\mathbb{D}$, are diagonal. $\mathbf{\Gamma}$ and $\mathbf{F}$ are respectively the torques and forces which act on the bubble due to non-zero viscosity, and the presence of vorticity in the flow, as well as buoyancy. The forces include buoyancy, drag, and wake-induced lift, while the torques include drag and wake-induced torque,

$$
\mathbf{F}=\mathbf{F}_{\mathrm{B}}+\mathbf{F}_{\mathrm{D}}+\mathbf{F}_{\mathrm{L}} \quad \text { and } \quad \boldsymbol{\Gamma}=\boldsymbol{\Gamma}_{\mathrm{D}}+\boldsymbol{\Gamma}_{\mathrm{W}}
$$

The buoyancy force $\mathbf{F}_{\mathrm{B}}=\rho V \mathbf{g}$ is vertical and, hence, generally has components in the 1- and 2-directions, say $F_{\mathrm{B} 1}$ and $F_{\mathrm{B} 2}$. The partition between the 1- and 2-components is determined by the pitch angle $\theta$ between the path and the vertical. Here $V$ is the volume of the bubble and $\mathbf{g}$ is the acceleration due to gravity. The density $\rho$ is actually the density difference between water and air, but since this difference is vast, we replace $\rho_{\text {water }}-\rho_{\text {air }}$ with $\rho_{\text {water }}$ and drop the subscript for brevity. The drag force is defined as $F_{\mathrm{D}}=0.5 C_{\mathrm{D}} \pi R^{2} \rho U^{2}$. The drag coefficient $C_{\mathrm{D}}$ is a nontrivial function of bubble shape and Reynolds number. The direction of drag is by definition opposite to the velocity. For a straight rising bubble, Moore's analytical result [12] successfully predicts our measurements of $C_{\mathrm{D}}$ as well as those of other experiments [13] (provided the bubble shape is known). Moore's result is

$$
C_{\mathrm{D}}=\frac{48}{R e} G(\chi)+\frac{48}{R e^{3 / 2}} G(\chi) H(\chi) .
$$

In the first term on the right, 48/Re results from computing the dissipation in the flow field predicted by potential flow theory for a free-slip sphere. $G(\chi)$ corrects for the fact that the bubble is an ellipsoid rather than a sphere. The second term on the right estimates the contribution to drag due to rotational flow in thin boundary layers and a long thin wake and is one to two orders of magnitude smaller than the first term. Once the path and wake become unstable, Moore's prediction is no longer expected to be accurate for two reasons. First, the rotational regions of the flow change drastically when the wake 
goes unstable and loses axisymmetry. Second, the first term on the right in equation (5) is based on the irrotational flow around a translating body and a different result is expected for a body with some rotation as well. This difference may be small since the timescale of rotation (path oscillation period) is about $200 \mathrm{~ms}$, which is large compared to timescale for translation, $D / U \approx 10 \mathrm{~ms}$.

For the purposes of the comparisons in the next section, we concern ourselves only with straight rising or spiralling bubbles. From equations (2) and (3) one may derive the equations of a spiral,

$$
\begin{aligned}
& \Gamma_{\mathrm{W} 1}=\Gamma_{\mathrm{D} 1}, \\
& \Gamma_{\mathrm{W} 2}=\Gamma_{\mathrm{D} 2}, \\
& F_{\mathrm{D}}=F_{\mathrm{B}} \cos \theta, \\
& F_{\mathrm{L} 2}=F_{\mathrm{B}} \sin \theta, \\
& F_{\mathrm{L} 3}=\rho V\left(C_{M 3} a_{3}+C_{M 1} 2 \pi f \sin \theta U\right) .
\end{aligned}
$$

Here $C_{M i}$ are the added mass coefficients of an oblate ellipsoid, $a_{3}$ is the acceleration component in the 3 -direction, and $f$ is the frequency of path oscillations. For a straight rising bubble the situation is much simpler, requiring only one equation,

$$
0=F_{\mathrm{B} 1}+F_{\mathrm{D}} .
$$

\section{Experimental observations}

In this section we present observations of the behaviour of bubbles rising in a $1 \%$ polyacrylamide/water solution and compare these dynamics to those of bubbles in pure water.

Small bubbles. First, we observe a difference in the rise behaviour of very small bubbles, which rise rectilinearly through solutions of different polymer concentration. Here, the equivalent bubble radius is $0.64 \mathrm{~mm}$ (at $1 \mathrm{~atm}$ ), which is significantly below the critical size for path instability. As demonstrated in figure 5, a bubble rising in pure water (trace (a) in figure 5) quickly achieves its terminal velocity and maintains this speed indefinitely. For increasing polymer concentration, reaching terminal speed is obviously a different process. During a rise of about $0.5 \mathrm{~m}$, these bubbles show no sign of reaching a terminal speed; instead, the velocity steadily drops. We note that polyacrylamide is not known to be a surface active molecule.

Onset. One of the most dramatic changes in observed dynamics is the shift of the onset of path instability to smaller bubble size. As a measure of the onset, figure 6 shows the horizontal component of velocity averaged between 1.4 and $1.5 \mathrm{~m}$ above the release point for varying bubble size. The horizontal velocity is zero for a straight rising bubble. In terms of Reynolds number, the onset is $700 \pm 5$ for water and $570 \pm 5$ for the polymer solution: a $20 \%$ shift. Furthermore, it appears that the bifurcation to path instability for increasing bubble size is less abrupt for the polymer case. In previous work it has been suggested that this bifurcation may be subcritical in water $[2,8]$. Our 


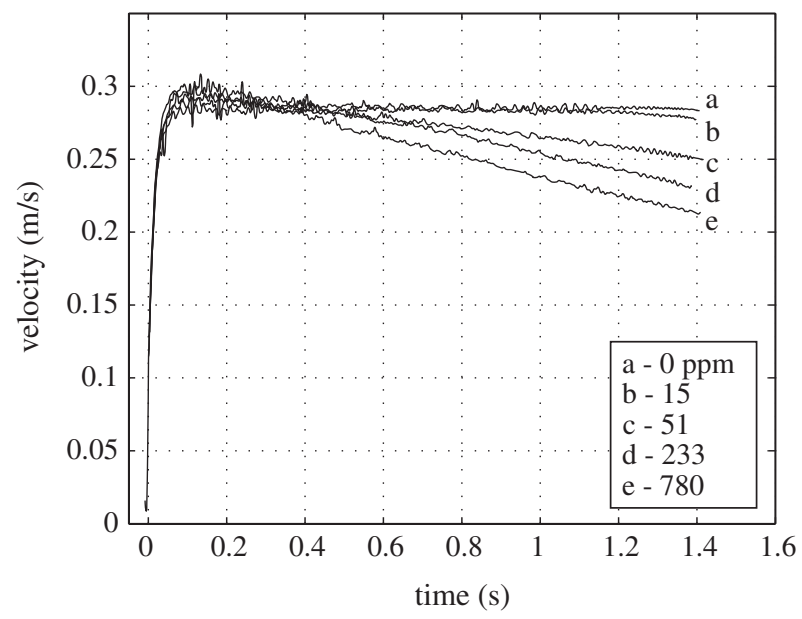

Figure 5. As the concentration of polyacrylamide is increased the bubble is slower in reaching a steady terminal velocity.

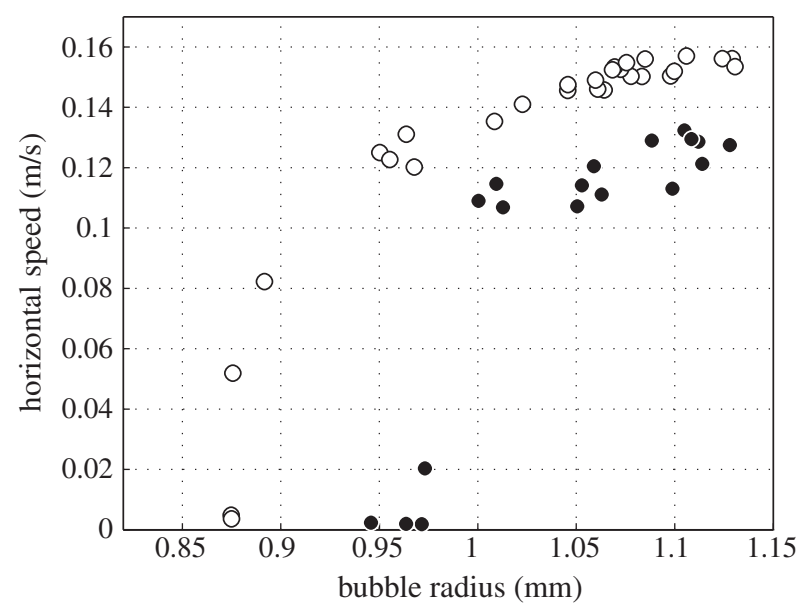

Figure 6. The critical bubble size for which path instability occurs is decreased in the polymer solution (open circles) compared to water (solid circles).

observations support this claim for water, but the more gradual bifurcation with polymer added suggests a possible supercritical transition in this case.

Speed and path geometry. After the path becomes unstable, we may compare the bubble velocity and the geometrical features of the oscillatory trajectory. We avoid the complicated unsteady force dynamics during the zigzag and restrict comparisons to bubbles in a fully developed spiral state. As such, the velocity magnitudes are close to constant. Figure 7 displays the total velocity, vertical component of velocity, horizontal component of velocity, pitch angle of the spiral, as well as the frequency and diameter of the spiral. A spiralling bubble rising in water moves with a higher speed than that in the polymer solution. Although the horizontal component of velocity is larger for the polymer solution, the vertical velocity component is sufficiently large in water to result in a larger total velocity. Since the frequency of spiral motion is nearly equal for both water and the polymer solution, the larger spiral diameter for the polymer solution is consistent 
Viscoelastic effects on the dynamics of a rising bubble
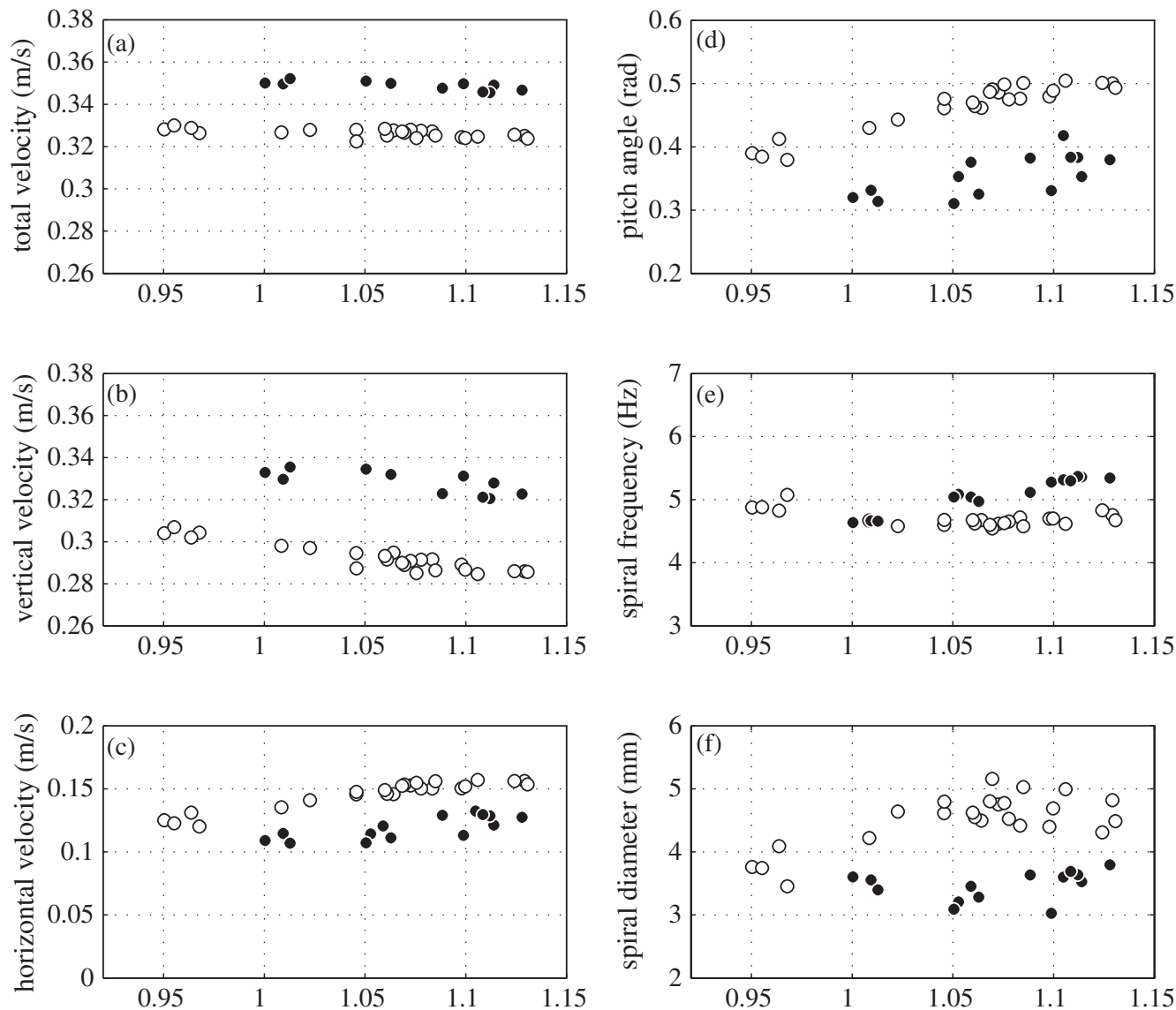

Figure 7. As reflected in the vertical velocity component (b), the total velocity (a) for a given bubble is smaller for the polymer solution (open circles), in spite of the fact that the horizontal velocity component (c) is smaller for water (solid circles). The pitch angle (d) and diameter (f) of the spiral are larger for the polymer solution, while the frequency of spiral oscillations (e) is comparable for water and polymer. All properties are measured during the fully developed spiral state.

with the fact that the horizontal velocity is also larger. The pitch angle is important since it determines the fraction of the buoyancy force, which is aligned with the velocity, i.e. the fraction available to drive the bubble forward. When the pitch angle is large, less buoyancy is available to propel the bubble ahead. The remaining fraction of buoyancy must be balanced by the lift.

Forces on straight rising bubbles. Let us now compare some of the forces which influence the dynamics described above. Beginning with the simplest, buoyancy is obviously equal for equal bubble size. For straight rising bubbles, only drag may balance the buoyancy. Therefore, drag is also equal for equal bubble size in the straight rise case. Nonetheless, the rise velocities are different for equal sized bubbles rising rectilinearly through water and the polymer solution. This reflects the fact that the drag coefficient is different. Moore's prediction (equation (5)) for the drag coefficient depends on the Reynolds number, and hence viscosity, as well as the aspect ratio of the bubble. In addition, a priori, one might suppose that the drag coefficient also depends on elastic 

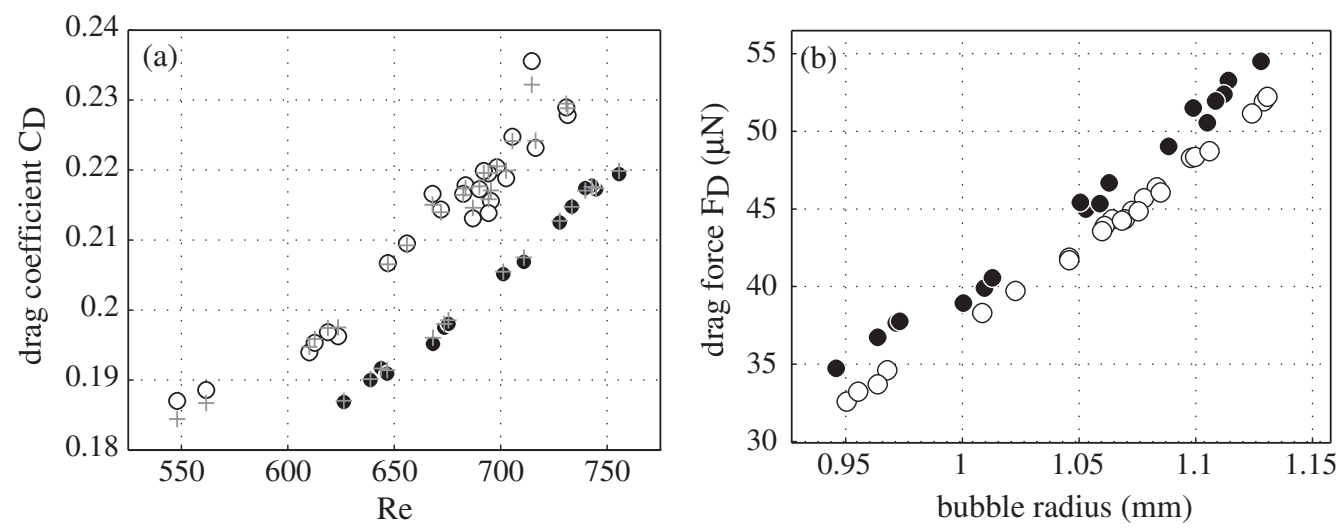

Figure 8. (a) During the rectilinear portion of the bubble trajectories, our drag coefficient measurements are in agreement with Moore's theory (+). (b) The measured drag force for spiralling bubbles in the polymer solution (open circles) is less than that in water (solid circles).

fluid properties for a bubble in the polymer solution. As demonstrated in figure 8, we find that the change in the $C_{\mathrm{D}}$ versus $R e$ curve is well predicted by accounting only for the viscosity change (due to temperature as well as fluid properties). This result strongly suggests that the aspect ratio of a bubble in the polymer solution is the same as that of an equal sized bubble in water. This result facilitates some later analysis, which uses the aspect ratio. Furthermore, the result suggests that, for this polymer solution, the elastic properties of the fluid have negligible effects on $C_{\mathrm{D}}$.

Forces on spiralling bubbles. The drag measured during the spiral is different for bubbles rising in water compared to the polymer solution as shown in figure 8(b). This is reflected directly in the difference in pitch angle between bubble trajectories in the two test fluids (see figure $7(\mathrm{~d})$ ). The pitch angle determines the fraction of buoyancy which must be balanced by drag so as to maintain a constant velocity. Naturally, agreement with Moore's theory does not persist once the bubble's path and wake become unstable, since the correction due to rotational flow must be different, as discussed in section 4 . We find that the drag coefficient of spiralling bubbles is slightly lower (1-2\%) than the value predicted based on just the dissipation in the irrotational flow (i.e. $48 G(\chi) / R e$ ). The difference between measured drag and potential flow drag was slightly greater for polymer than for water.

Finally, we compare measurements of lift forces. Our measurement methods for lift are independent of viscosity and, for the 2-component of lift $F_{\mathrm{L} 2}$, independent of aspect ratio as well. As described in the previous section, $F_{\mathrm{L} 2}$ is balanced only by the 2-component of buoyancy $F_{\mathrm{B} 2}$ for a spiralling bubble. The magnitude of $F_{\mathrm{B} 2}$ is yielded directly from our measurements of pitch angle. It is evident in figure $9\left(\right.$ a) that $F_{\mathrm{L} 2}$ is greater for bubbles in the polymer solution.

The remaining fraction of the lift force $F_{\mathrm{L} 3}$ is obtained from measurements of the bubble speed, acceleration, pitch angle, and shape (see equation (6)). $F_{\mathrm{L} 3}$ is a measure of the centrifugal force which maintains the circular nature of the spiral and the rotation about the bubble centre of mass. This measurement, shown in figure 9(b), confirms that lift forces are stronger for bubbles in the polymer solution. 

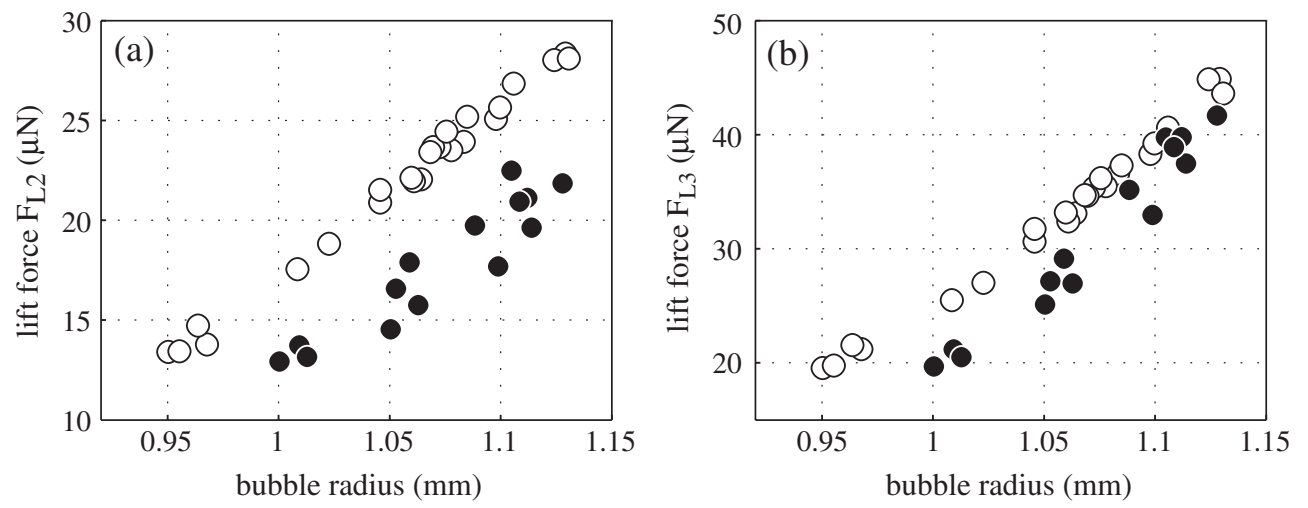

Figure 9. The lift forces which balance buoyancy (a) and produce the curvature of the spiral (b) are both greater for the polymer solution (open circles) compared to water (solid circles).

\section{Summary and discussion}

We compare the behaviour of millimetre-sized bubbles rising in water to that in a viscoelastic polymer solution. The Deborah number for the flow around our bubbles is near unity and the first normal stress difference is non-zero. In such conditions purely elastic fluid instabilities are known to exist at arbitrarily low Re, particularly when streamlines are curved (e.g. $[33,29,34,35])$. Nonetheless, we remind the reader that our experiment is different from typical studies of viscoelastic flows because the viscosity is quite low and the Reynolds number quite large, $R e \sim 1000$. The result is that the fluid instability in the bubble's wake remains qualitatively similar to that observed in Newtonian fluids. The viscoelastic effects are evident in quantitative changes in the onset of wake instability, path geometry, and measured lift forces.

The onset of the transition to path and wake instability occurs for smaller bubbles in the polymer solution than in water. The viscosity of the polymer solution is greater than the viscosity of water. An increase in viscosity in a Newtonian fluid would give rise to quite the opposite shift in the onset.

In spite of slower rise speeds and higher viscosity of the polymer solution, our measurements indicate that the flow in the bubble's wake is more intense in the polymer solution. This is most clearly apparent in our precise measurements of lift force. The magnitude of the lift forces may be related directly to the momentum transfer normal to the trajectory due to fluid pumping of the wake. Stronger wake vortices imply a larger momentum transfer, which in turn implies larger lift forces. Indeed, our measurements of lift forces indicate a $25 \%$ increase in the polymer solution compared to water.

Further evidence of more intense flow in the polymer fluid wake may be deduced from the energetics of a rising bubble. As a bubble rises, buoyancy is ultimately the only source of energy. The integrated work done by buoyancy over the bubble's entire ascent is a direct measure of the total increase in energy of the fluid. This quantity is obviously equal for equal sized bubbles independent of fluid properties as well as speed and geometry of the bubble's path. On the other hand, the rate at which energy is delivered to the fluid depends on the bubble's speed, $\Phi_{\mathrm{TO}}=\mathbf{F}_{\mathrm{B}} \cdot \mathbf{u}=F_{\mathrm{B}} u_{z}$. Since $u_{z}$ is smaller in the 
polymer solution for a given bubble size, the power delivered to the fluid is smaller. Let us explore the implications of the power budget on the state of the bubble's wake. The net power delivered to the fluid at any given instant may be divided in to two contributions, $\Phi_{\text {TOTAL }}=\Phi_{\mathrm{p}}+\Phi_{\mathrm{r}}$, where $\Phi_{\mathrm{p}}$ is the energy dissipated in the irrotational parts of the flow and $\Phi_{\mathrm{r}}$ accounts for that in the rotational regions of flow. Analytical calculations suggest that $\Phi_{\mathrm{p}}$ is proportional to $U^{2}$ and accounts for the majority of the net power $\Phi_{\text {TотAL }}$ (e.g. [12]). When the bubble's path becomes unstable, the velocity drops, say by $\Delta U$. This drop is larger for bubbles in the polymer solution than for those in water. The resulting drop in net instantaneous power delivery $\Delta \Phi_{\text {ТОТАL }}=\mathbf{F}_{\mathrm{B}} \cdot \mathbf{u} \sim \Delta U$ is smaller than the drop $\Delta \Phi_{\mathrm{p}} \sim(\Delta U)^{2}$, which implies that $\Phi_{\mathrm{r}}$ must increase to compensate. In this sense, the reduction in $v_{z}$ observed for bubbles in the polymer solution compared to those in water provides evidence for increased power consumption in the rotational parts of the flow. This observation confirms the existence of more intense flow structures in the wake as suggested by the lift force measurements.

The observation that small bubbles tend to gradually slow down after an initial acceleration period remains mysterious and may benefit from additional investigation. In addition, it may be interesting to measure the drag on rather large bubbles, say $R e \sim 10^{3}-10^{4}$. For such $R e$, rigid bodies with no-slip boundaries are known to exhibit drag reduction in elastic fluids compared to Newtonian fluids. The free-slip boundary of a bubble would allow one to probe the influence of viscous boundary layers in these effects.

\section{Acknowledgments}

The development of the ideas in this paper benefitted from many discussions at the Euromech colloquium 465, 'Hydrodynamics of bubbly flows'. We also appreciate the skilled work of Denis Le Tourneau and Pascal Metz in building the water tank and the ultrasound device respectively. This work was funded by École Normale Supérieur, Centre National de la Recherche Scientifique, and Region Rhone-Alpes, Emergence 1462.

\section{References}

[1] Prosperetti A, Bubbles, 2004 Phys. Fluids 161852

[2] Shew W L, Poncet S and Pinton J F, Path instability and wake of a rising bubble, 2005 Phys. Fluids submitted, available at http://hal.ccsd.fr/ccsd-00013378

[3] Brücker C, Structure and dynamics of the wake of bubbles and its relevance for bubble interaction, 1999 Phys. Fluids 111781

[4] de Vries A W G, Path and wake of a rising bubble, 2001 PhD Dissertation University of Twente, Netherlands

[5] de Vries A W G, Biesheuvel A and van Wijngaarden L, Notes on the path and wake of a gas bubble rising in pure water, 2002 Int. J. Multiph. Flow 281823

[6] Ellingsen K and Risso F, On the rise of an ellipsoidal bubble in water: oscillatory paths and liquid-induced velocity, 2001 J. Fluid Mech. 440235

[7] Lunde K and Perkins R J, Observations on wakes behind spheroidal bubbles and particles, 1997 Paper No. FEDSM'97-3530, 1997 ASMEFED Summer Meeting (Vancouver, Canada)

[8] Mougin G and Magnaudet J, Path instability of a rising bubble, 2002 Phys. Rev. Lett. 88014502

[9] Mougin G, Interactions entre la dynamique d'une bulle et les instabilitiés de son sillage, $2002 \mathrm{PhD}$ Dissertation Institute National Polytechnique de Toulouse, France

[10] Veldhuis C, 2005 personal communications

[11] Wu M and Gharib M, Experimental studies on the shape and path of small air bubbles rising in clean water, 2002 Phys. Fluids 14 L49 
Viscoelastic effects on the dynamics of a rising bubble

[12] Moore D W, The velocity of rise of distorted gas bubbles in a liquid of small viscosity, 1965 J. Fluid Mech. 23749

[13] Duineveld P C, The rise velocity and shape of bubbles in pure water at high Reynolds number, 1995 J. Fluid Mech. 292325

[14] Clift R, Grace J R and Weber M E, 1978 Bubbles, Drops, and Particles (New York: Academic)

[15] Aybers N M and Tapucu A, The motion of gas bubbles rising through stagnant liquid, 1969 Wärme-Stoffübertrag. 2118

[16] Aybers N M and Tapucu A, Studies on the drag and shape of gas bubbles rising through stagnant liquid, 1969 Wärme-Stoffübertrag. 2171

[17] Benjamin T B, Hamiltonian theory for motions of bubbles in an infinite liquid, 1984 J. Fluid Mech. 181349

[18] Hartunian R A and Sears W R, On the instability of small gas bubbles moving uniformly in various liquids, 1957 J. Fluid Mech. 327

[19] Leifer I, Patro R K and Bowyer P, A study on the temperature variation of rise velocity for large clean bubbles, 2000 J. Atmos. Ocean. Technol. 171392

[20] Magnaudet J and Eames I, The motion of high-Reynolds-number bubbles in inhomogeneous flows, 2000 Ann. Rev. Fluid Mech. 32659

[21] Mercier J, Lyrio A and Forslund R, Three dimensional study of the nonrectilinear trajectory of air bubbles rising in water, 1973 J. Appl. Mech. 40650

[22] Saffman P G, On the rise of small air bubbles in water, 1956 J. Fluid Mech. 1249

[23] Mordant N, Pinton J F and Michel O, Time-resolved tracking of a sound scatterer in a complex flow: nonstationary signal analysis and applications, 2002 J. Acoust. Soc. Am. 112108

[24] Mordant N and Pinton J F, Velocity measurements of a settling sphere, 2000 Eur. Phys. J. B 18343

[25] Mordant N, Metz P, Michel O and Pinton J F, An acoustic technique for Lagrangian velocity measurements, 2005 Rev. Sci. Instrum. 76025105

[26] Walters K, 1975 Rheometry (London: Chapman and Hall)

[27] Boger D V, Viscoelastic fluid mechanics: interaction between prediction and experiment, 1996 Exp. Thermal Fluid Sci. 12234

[28] Argumedo A, Tung T T and Chang K I, Rheological property measurements of drag-reducing polyacrylamide solutions, 1978 J. Rheol. 22449

[29] Boger D and Walters K, 1993 Rheological Phenomena in Focus (Amsterdam: Elsevier)

[30] Lamb H, 1945 Hydrodynamics 6th edn (New York: Dover)

[31] Howe M S, On the force and moment on a body in an incompressible fluid, with application to rigid bodies and bubbles at low and high Reynolds numbers, 1995 Q. J. Mech. Appl. Math. 48401

[32] Mougin G and Magnaudet J, The generalized Kirchhoff equations and their application to the interaction between a rigid body and an arbitrary time-dependent viscous flow, 2002 Int. J. Mulitph. Flow 281837

[33] Shaqfeh E S G, Purely elastic instabilities in viscoelastic flows, 1996 Ann. Rev. Fluid Mech. 31129

[34] Groisman A and Steinberg V, Mechanism of elastic instability in Couette flow of polymer solutions: experiment, 1998 Phys. Fluids 102541

[35] McKinley G H, Armstrong R C and Brown R A, The wake instability in viscoelastic flow past confined circular cylinders, 1993 Phil. Trans.: Phys. Sci. Eng. 344265 